\title{
Associations of Embeddedness and Posttraumatic Stress Disorder among 9/11 Survivors
}

\author{
Meghan K. Hamwey*D, Cristina D. Pollari, Sukhminder Osahan, Sascha K. Garrey, Felix M. Ortega, \\ Adrienne Solomon and Robert M. Brackbill
}

World Trade Center Health Registry, New York City Department of Health and Mental Hygiene, Long Island City, NY 11101, USA; cpollari@health.nyc.gov (C.D.P.); osukhmin@health.nyc.gov (S.O.); sgarrey@health.nyc.gov (S.K.G.); fortega@health.nyc.gov (F.M.O.); asolomon1@health.nyc.gov (A.S.); rbrackbi@health.nyc.gov (R.M.B.)

* Correspondence: mhamwey@health.nyc.gov

Citation: Hamwey, M.K.; Pollari, C.D.; Osahan, S.; Garrey, S.K.; Ortega, F.M.; Solomon, A.; Brackbill, R.M. Associations of Embeddedness and Posttraumatic Stress Disorder among 9/11 Survivors. Epidemiologia 2021, 2, 608-620. https://doi.org/10.3390/ epidemiologia2040041

Academic Editor: Janice Hegewald

Received: 27 September 2021

Accepted: 2 December 2021

Published: 8 December 2021

Publisher's Note: MDPI stays neutral with regard to jurisdictional claims in published maps and institutional affiliations.

Copyright: (c) 2021 by the authors. Licensee MDPI, Basel, Switzerland. This article is an open access article distributed under the terms and conditions of the Creative Commons Attribution (CC BY) license (https:/ / creativecommons.org/licenses/by/ $4.0 /)$.

\begin{abstract}
Following exposures to traumatic events on 9/11, survivors have reported heightened levels of posttraumatic stress disorder (PTSD). Multiple factors contribute to both the exacerbation and amelioration of PTSD symptoms, including social integration and support. This cross-sectional study aimed to understand and identify associations of embeddedness and psychosocial risk factors by PTSD status for survivors and first responders of $9 / 11$. Results indicate that those with chronic PTSD had the lowest prevalence of both social and emotional embeddedness and many who reported no PTSD symptoms following 9/11 reported moderate levels of social and emotional embeddedness. Overall, our findings suggest those individuals who reported little to no PTSD also reported the most social/emotional embeddedness; whereas those individuals who report greater or chronic PTSD report the least social/emotional embeddedness. As such, it may be beneficial for clinicians across multiple care disciplines and contexts to consider and address the social lives and needs of those individuals experiencing symptoms of PTSD to ensure their emotional and physical needs are truly being met.
\end{abstract}

Keywords: embeddedness; PTSD; 9/11; social support; self-efficacy; social integration

\section{Introduction}

Post-traumatic stress disorder (PTSD) is the most prevalent mental health outcome among individuals directly and indirectly exposed to the terrorist attacks on 11 September 2001 (9/11) [1-3], and particularly among those who experienced trauma (e.g., injury, loss of a loved one) [4-6]. There are multiple mechanisms associated with PTSD (e.g., emotional engagement, stress tolerance, extinction, negative post-traumatic experiences), most of which tend to be driven by one's psychology neurobiology, epigenetics, and/or biology. One of the psychological implications of PTSD is the avoidance of participation in social life, such as going to work or engaging in leisure activities. As a result of PTSD-related social withdrawal, there is often an increased and problematic use of substances such as alcohol, illicit drugs, and other substances with psychoactive properties [6-8]. A lack of social support and social integration have also been shown to be related to long-term PTSD $[6,9,10]$. The amelioration of PTSD symptoms would thus be bolstered by increased social support and social integration, or perhaps therapeutic intervention.

Social isolation, or the lack of social contact via in person or other means, is a characteristic feature of, experiencing PTSD symptoms and it can detrimentally affect individuals of all ages emotionally, physically, and mentally [11]. It comprises an objective deficit in both the quality and quantity of social ties and interactions with other individuals or a person's wider community [12]. Further, social isolation has been found to be associated with a number of negative health outcomes, including an increased risk of mortality in older adults [13], depression [14], and suicidal ideation [15,16]. Loneliness, defined as the 
subjective feeling of a discrepancy between one's desired and actual relationships, is an emotionally driven consequence of social isolation [17]. As with social isolation, loneliness is similarly linked to negative health outcomes, including mortality [18] and depression [18]. However, the literature is inconclusive on how the social dimensions of social isolation and the emotional dimensions of loneliness relate to their shared health risks.

There is a growing body of literature that explores the concept of social and emotional embeddedness, which is the opposite of loneliness, in the context of individual demographic groups. For instance, Snowden and colleagues [19] found that, in a general sample of the United States population, African American men reported higher levels of overall social embeddedness than White men and that social involvement was an important factor in predicting psychological well-being. Another study that considered older Chinese adults living in rural areas, found that social embeddedness was positively associated with health information and help-seeking behavior via its status as a bolsterer of health-sharing behavior in this population [20]. Embeddedness further reflects both the size of social ties one has within their family and the greater community, as well as how connected one feels within their social network, irrespective of its size [21,22].

The presence or development of embeddedness could positively influence the course of PTSD. One factor that may contribute to greater embeddedness among individuals experiencing PTSD symptoms is individual self-efficacy, which signifies an individual's belief in their personal capacity to perform in specific manners (e.g., social interactions). For instance, higher levels of coping self-efficacy have been found to have a buffering effect on PTSD symptoms and promote PTSD recovery $[23,24]$. Social integration, or the presence of social ties that can provide help and support to an individual's perceived social support, or the subjective belief of how integrated one is in their social network, have also been found to be associated with lower levels of PTSD symptomology that result after exposure to natural disasters [25] and as potential sources for reduced stress among veterans [10]. In turn, we postulate that social and emotional embeddedness would have an association with the persistence of PTSD over time. Studies have categorized the course of PTSD since the 9/11-initiating event into trajectories that include resistant, delayed onset, recovering, or chronic [26]. Trajectories that remain high or chronic, that are refractory to the improvement of symptoms, are associated with low social support and 9/11-related unemployment [27]. It could be surmised that increased social and emotional embeddedness would lessen the persistence of PTSD and perhaps increase the effectiveness of treatment or be a treatment goal.

\section{Study Aims}

Data from the World Trade Center Health Registry were used to explore the relationship between social and emotional embeddedness and the course of PTSD among persons directly exposed to the $9 / 11$ attacks. We aim to: (1) describe the prevalence of social and emotional embeddedness among persons directly exposed to the $9 / 11$ attacks, 15 years after their exposure, across four PTSD groups (i.e., no PTSD or resistant, delayed, recovered, and chronic), and (2) examine the associations of demographic factors and psychosocial characteristics (i.e., social support, social integration, and self-efficacy) with a new calculation of social and emotional embeddedness, stratified by probable 9/11-related PTSD status.

\section{Materials and Methods}

The World Trade Center Health Registry (WTCHR) was established by the New York City Department of Health and Mental Hygiene with the goal of monitoring the physical and mental health effects of those individuals exposed to the attacks on 9/11. The WTCHR is a longitudinal cohort study comprising 71,426 enrollees at the first survey wave (2003-2004). An additional three survey waves have also been collected in 2006-2007 (Wave 2), 2010-2011 (Wave 3), and 2015-2016 (Wave 4). The enrollees are comprised of rescue and recovery workers, lower Manhattan residents, local workers, school students 
and staff, and occupants/passersby on 9/11. Details of recruitment and data collection are published elsewhere [28].

The analytic sample for this study was restricted to enrollees who participated in the Health and Quality of Life (HQoL) 15 years after the 9/11 survey, which was an in-depth study of a subset of rescue and recovery workers and survivors who indicated they had sustained injuries on $9 / 11$ and a comparison group of non-injured persons $(n=6544)$. The sample size for the study included all persons who reported being injured on 9/11/2001 and a 3 to 1 comparison group. Eligibility criteria included completing all four survey waves, being $\geq 18$ years old at the time of collection, and English speaking. Enrollees who had missing data on the PTSD Checklist-Specific at any of the four survey waves were excluded from the study $(n=704)$. The final analytic sample resulted in 5840 enrollees. Importantly, the HQoL survey was only conducted at one time point, thus the present study is cross-sectional in design and analysis.

The WTCHR protocol was approved by the Institutional Review Boards at the Centers for Disease Control and Prevention and the New York City Department of Health and Mental Hygiene. Oral informed consent was obtained from participants at WTCHR enrollment using computer-assisted telephone interviews (CATI). This consent was in addition to the implicit consent by enrollees who completed each subsequent follow-up survey, as well as for the HQoL survey. Enrollees were regularly reminded by letter of their rights associated with being enrolled in the Registry, including the options of withdrawing from the Registry or not responding to follow-up surveys.

\subsection{Measures}

Demographics. Age collected at the time of the HQoL (ranges: <50, 50-59, 60-64, or $\geq 65$ years old), sex collected at Wave 1 (male or female), race/ethnicity collected at Wave 1 (non-Hispanic White, non-Hispanic Black, Hispanic/Latino, Asian, or multiracial/other), Wave 4 employment (employed or not employed), and marital status (married/living with partner consistently across 4 survey waves, or not) were all included as covariates in this study.

Social and emotional embeddedness were measured using the De Jong Gierveld (2006) 6-item loneliness questionnaire [29] during the HQoL survey only and thus largely post-PTSD measures. The loneliness questionnaire contains three items specific to social loneliness and three for emotional loneliness. Item responses were categorical with three levels, "yes", "more or less", and "no". Social and emotional loneliness scores were inverted to construct the embeddedness measure.

Social embeddedness. Social loneliness items were used to derive a social embeddedness construct. Items included "There are plenty of people I can rely on when I have problems", "There are many people I can trust completely", and "There are enough people I feel close to". The social items were positively worded, and each response received the following reversescored value, yes $=0$, more or less $=1$, and no $=1$. Items were summed to get a social loneliness total score, ranging from 0 to 3 . Scores were not calculated when item responses were missing $(n=170)$. Social embeddedness was calculated by having a social loneliness total score of $\leq 1$, that is those who indicated low levels of social loneliness were assigned a high level of embeddedness.

Emotional embeddedness. Emotional loneliness items were used to derive an emotional embeddedness construct. Items included "I experience a general sense of emptiness", "I miss having people around", and "I often feel rejected". All emotional items were negatively worded and coded as yes $=1$, more or less $=1$, and no $=0$ (Note that emotional and social embeddedness are reversed on direction of answer to questions). The items were summed to obtain a total emotional loneliness score, with scores ranging from 0 to 3 . Scores were not calculated when item responses were missing $(n=174)$. Emotional embeddedness was calculated by having an emotional loneliness total score of $\leq 1$. The thresholds for emotional and social embeddedness were derived from those used by De Jong Gierveld loneliness questionnaire scoring. 
Post-traumatic stress disorder. Probable post-9/11 PTSD was evaluated across all four survey waves using the PTSD Checklist (PCL) 17-item questionnaire [30,31]. The PCL is a self-administered scale that assesses PTSD symptoms based on DSM-IV criteria [32]. Items focused on re-experiencing symptoms specific to $9 / 11$ in the past 30 days, with responses ranging from $1=$ not at all to $5=$ extremely. A threshold of a PCL total score of 44 or greater indicated probable PTSD with a reported sensitivity of 0.78 and a specificity of 0.97 (see Ruggiero et al.).

Using the PCL total score, probable PTSD was organized into four different categories: chronic, delayed, recovered, and no PTSD. Those with no PTSD (resilient) were defined as having PCL total scores $<44$ across all 4 survey waves. Chronic PTSD was defined as having PCL total scores $\geq 44$ across all 4 survey waves. Delayed PTSD was considered as having PCL total scores $<44$ at Waves 1 and 2 and PCL total scores $\geq 44$ at Waves 3 and 4 . Lastly, the recovered group was expressed as PCL total scores $\geq 44$ at Waves 1 and 2 and PCL total scores $<44$ at Waves 3 and 4 .

Social integration. Social integration was measured using four items adapted from the RAND Social Health Battery [33]. Participants were asked to indicate whether they have experienced the following in the last 30 days: "having 1 or more close friends", "having visited/talked to/emailed friends at least twice in the last 30 days", "attended a religious service at least twice in last 30 days", or "been actively involved in a volunteer organization or club in last 30 days". The number of endorsed items were summed and categorized using three levels: low (0-1), medium (2), and high ( $\geq 3)$. Prior use of these categories of social integration provided a clear dose-response relationship with various outcomes including depression.

Social support. Social support was measured at Wave 4 using the Social Support Survey for the Medical Outcomes Study, 5-item version [34]. Items asked participants how often someone was available to perform the following tasks for them: "have a good time with", "hug you", "take you to the doctor", "prepare your meals if you are unable", and "understand your problems". Responses ranged from $0=$ none of the time to $4=$ all of the time. The five items were summed with total scores ranging from 0-20. Social support was categorized as low $\leq 10$, medium 11-15, and high as 16-20, similarly to social integration categories.

Self-efficacy. Self-efficacy was collected at Wave 4 using the General Self-Efficacy Scale (GSE) [35], which included five items measured on a four-point scale. The items were "It is easy for me to stick to my aims and accomplish my goals", "I am confident that I could deal efficiently with unexpected events", "Thanks to my resourcefulness, I know how to handle unforeseen situations", "I can remain calm when facing difficulties because I can rely on my coping abilities", and "No matter what comes my way, I am usually able to handle it". Responses ranged from $0=$ not at all true to 4 = exactly true. Items were summed to create a total score ranging from 0-20. Low self-efficacy was defined as having a total score $<17$ and high self-efficacy as $\geq 17$, based on the median score for the entire Registry sample.

\subsection{Analytic Strategy}

Overall frequencies of demographic characteristics and psycho-social variables were calculated. Bivariate analyses were conducted for demographic characteristics and psychosocial variables with social and emotional embeddedness, using unadjusted odds ratios and $95 \%$ confidence intervals.

Multivariable and Log-linear regression models were used to examine the association of the psycho-social variables with social and emotional embeddedness, separately. Each model was adjusted for age, race, sex, and stratified by PTSD group (chronic, delayed, recovered, no PTSD). Odds ratios and 95\% confidence intervals were computed. The significance level was set at a 2-sided value of alpha $<0.05$ to identify plausible relationships between PTSD history and social and emotional embeddedness recognizing that multiple comparisons may yield associations by chance that are not statistically significant at this level.

All analyses were conducted using SAS 9.4 (SAS Institute, Inc., Cary, North Carolina). 


\section{Results}

\subsection{Demographic Characteristics}

A total of 5840 enrollees were included in this sample. On average, enrollees were 58 years old $(M=58.1 ; S D=10.2)$. The majority of enrollees were male $(64.9 \%)$ and White $(78.6 \%)$. Further, the majority of enrollees indicated they were employed $(57.5 \%)$ and married/living with a partner (58.7\%) (not shown in tables).

\subsection{Social Embeddedness}

Table 1 shows the distribution of demographic factors and psycho-social factors with social embeddedness, stratified by PTSD group. The majority of enrollees never experienced PTSD $(n=4097)$ with a smaller portion falling into the other three PTSD groups. The delayed PTSD group was comprised of 309 enrollees, 406 were chronic, and 97 recovered over time. Overall, social embeddedness was more common among females $(46.3 \%)$, those younger than 50 years of age $(49.0 \%)$, those who were consistently married $(49.9 \%)$, non-Hispanic Whites $(47.9 \%)$, and those who indicated they were employed $(46.7 \%)$. Further, social embeddedness was more frequently reported among those with greater levels of social support (63.1\%), social integration (52.3\%), and self-efficacy (58.1\%). The highest prevalence of social embeddedness was found in the no PTSD group (52.2\%), followed by the recovered $(32.0 \%)$, delayed, $(26.2 \%)$, and chronic $(16.0 \%)$ groups.

Table 1. Distribution of demographic factors and psycho-social factors with social embeddedness.

\begin{tabular}{|c|c|c|c|c|c|c|c|c|c|c|c|c|c|c|c|}
\hline & \multicolumn{3}{|c|}{ All four PTSD Groups } & \multicolumn{3}{|c|}{ No PTSD Group } & \multicolumn{3}{|c|}{ Delayed PTSD Group } & \multicolumn{3}{|c|}{ Chronic PTSD Group } & \multicolumn{3}{|c|}{$\begin{array}{c}\text { Recovered PTSD } \\
\text { Group }\end{array}$} \\
\hline & No & Yes & Total & No & Yes & Total & No & Yes & Total & No & Yes & Total & No & Yes & Total \\
\hline & $\begin{array}{c}n= \\
3100\end{array}$ & $\begin{array}{c}n= \\
2570\end{array}$ & 5670 & $\begin{array}{c}n= \\
1959\end{array}$ & $\begin{array}{c}n= \\
2138\end{array}$ & 4097 & $\begin{array}{l}n= \\
228\end{array}$ & $\begin{array}{c}n= \\
81\end{array}$ & 309 & $\begin{array}{l}n= \\
341\end{array}$ & $\begin{array}{c}n= \\
65\end{array}$ & 406 & $\begin{array}{l}n= \\
66\end{array}$ & $\begin{array}{c}n= \\
31\end{array}$ & 97 \\
\hline Characteristic & $54.7 \%$ & $45.3 \%$ & & $47.8 \%$ & $52.2 \%$ & & $73.8 \%$ & $26.2 \%$ & & $84.0 \%$ & $16.0 \%$ & & $68.0 \%$ & $32.0 \%$ & \\
\hline \multicolumn{16}{|l|}{ Gender } \\
\hline \multirow[t]{2}{*}{ Male } & $\begin{array}{l}2036 \\
55.2\end{array}$ & $\begin{array}{l}1651 \\
44.8\end{array}$ & 3687 & $\begin{array}{l}1313 \\
48.4\end{array}$ & $\begin{array}{l}1402 \\
51.6\end{array}$ & 2715 & $\begin{array}{l}158 \\
72.8\end{array}$ & $\begin{array}{c}59 \\
272\end{array}$ & 217 & 198 & $\begin{array}{c}32 \\
139\end{array}$ & 230 & $\begin{array}{c}38 \\
691\end{array}$ & 17 & 55 \\
\hline & 55.2 & 44.8 & & 48.4 & 51.6 & & 72.8 & 27.2 & & 86.1 & 13.9 & & 69.1 & 30.9 & \\
\hline \multirow[t]{2}{*}{ Female } & 1064 & 919 & 1983 & 646 & 736 & 1382 & 70 & 22 & 92 & 143 & 33 & 176 & 28 & 14 & 42 \\
\hline & 53.7 & 46.3 & & 46.7 & 53.3 & & 76.1 & 23.9 & & 81.3 & 18.8 & & 66.7 & 33.3 & \\
\hline \multicolumn{16}{|l|}{$\begin{array}{l}\text { Age on Injury } \\
\text { Survey (years) }\end{array}$} \\
\hline \multirow[t]{2}{*}{$<50$} & 597 & 573 & 1170 & 388 & 480 & 868 & 48 & 24 & 72 & 53 & 8 & 61 & 12 & 5 & 17 \\
\hline & 51.0 & 49.0 & & 44.7 & 55.3 & & 66.7 & 33.3 & & 86.9 & 13.1 & & 70.6 & 29.4 & \\
\hline \multirow[t]{2}{*}{$50-59$} & 1075 & 791 & 1866 & 640 & 644 & 1284 & 98 & 25 & 123 & 134 & 28 & 162 & 24 & 13 & 37 \\
\hline & 57.6 & 42.4 & & 49.8 & 50.2 & & 79.7 & 20.3 & & 82.7 & 17.3 & & 64.9 & 35.1 & \\
\hline \multirow[t]{2}{*}{$60-64$} & 607 & 437 & 1044 & 372 & 356 & 728 & 42 & 15 & 57 & 68 & 10 & 78 & 19 & 6 & 25 \\
\hline & 58.1 & 41.9 & & 51.1 & 48.9 & & 73.7 & 26.3 & & 87.2 & 12.8 & & 76.0 & 24.0 & \\
\hline \multirow[t]{2}{*}{$65+$} & 821 & 769 & 1590 & 559 & 658 & 1217 & 40 & 17 & 57 & 86 & 19 & 10 & 11 & 7 & 18 \\
\hline & 51.6 & 48.4 & & 45.9 & 54.1 & & 70.2 & 29.8 & & 81.9 & 18.1 & & 61.1 & 38.9 & \\
\hline \multirow{2}{*}{\multicolumn{16}{|c|}{$\begin{array}{l}\text { Marital Status } \\
\text { W1-W4 }\end{array}$}} \\
\hline & & & & & & & & & & & & & & & \\
\hline \multirow[t]{2}{*}{ Always } & 1665 & 1658 & 3323 & 1101 & 1414 & 2515 & 121 & 50 & 171 & 150 & 38 & 188 & 30 & 17 & 47 \\
\hline & 50.1 & 49.9 & & 43.8 & 56.2 & & 70.8 & 29.2 & & 79.8 & 20.2 & & 63.8 & 36.2 & \\
\hline \multirow[t]{2}{*}{ Never/sometimes } & 1435 & 912 & 2347 & 858 & 724 & 1582 & 107 & 31 & 138 & 191 & 27 & 218 & 36 & 14 & 50 \\
\hline & 61.1 & 38.9 & & 54.2 & 45.8 & & 77.5 & 22.5 & & 87.6 & 12.4 & & 72.0 & 28.0 & \\
\hline \multicolumn{16}{|l|}{ Race/ethnicity } \\
\hline \multirow{2}{*}{$\begin{array}{l}\text { Non-Hispanic } \\
\text { White }\end{array}$} & 2323 & 2139 & 4462 & 1527 & 1799 & 3326 & 166 & 71 & 237 & 217 & 44 & 261 & 44 & 25 & 69 \\
\hline & 52.1 & 47.9 & & 45.9 & 54.1 & & 70.0 & 30.0 & & 83.1 & 16.9 & & 63.8 & 36.2 & \\
\hline \multirow{2}{*}{$\begin{array}{l}\text { Non-Hispanic } \\
\text { Black }\end{array}$} & 261 & 143 & 404 & 149 & 117 & 266 & 23 & 0 & 23 & 39 & 10 & 49 & 5 & 0 & 5 \\
\hline & 64.6 & 35.4 & & 56.0 & 44.0 & & 100.0 & 0.0 & & 79.6 & 20.4 & & 100.0 & 0.0 & \\
\hline \multirow[t]{2}{*}{ Hispanic } & 300 & 170 & 470 & 154 & 121 & 275 & 25 & 7 & 32 & 50 & 9 & 59 & 12 & 6 & 18 \\
\hline & 63.8 & 36.2 & & 56.0 & 44.0 & & 78.1 & 21.9 & & 84.8 & 15.3 & & 66.7 & 33.3 & \\
\hline \multirow[t]{2}{*}{ Asian } & 117 & 73 & 190 & 73 & 64 & 137 & 6 & 2 & 8 & 19 & 1 & 20 & 2 & 0 & 2 \\
\hline & 61.6 & 38.4 & & 53.3 & 46.7 & & 75.0 & 25.0 & & 95.0 & 5.0 & & 100.0 & 0.0 & \\
\hline Multiracial/other & 99 & 45 & 144 & 56 & 37 & 93 & 8 & 1 & 9 & 16 & 1 & 17 & 3 & 0 & 3 \\
\hline & 68.8 & 31.3 & & 60.2 & 39.8 & & 88.9 & 11.1 & & 94.1 & 5.9 & & 100.0 & 0.0 & \\
\hline Employment W4 & & & & & & & & & & & & & & & \\
\hline No & 1365 & 1048 & 2413 & 777 & 823 & 1600 & 110 & 45 & 155 & 217 & 39 & 256 & 23 & 14 & 37 \\
\hline & 56.6 & 43.4 & & 48.6 & 51.4 & & 71.0 & 29.0 & & 84.8 & 15.2 & & 62.2 & 37.8 & \\
\hline Yes & 1735 & 1522 & 3257 & 1182 & 1315 & 2497 & 118 & 36 & 154 & 124 & 26 & 150 & 43 & 17 & 60 \\
\hline & 53.3 & 46.7 & & 47.3 & 52.7 & & 76.6 & 23.4 & & 82.7 & 17.3 & & 71.7 & 28.3 & \\
\hline
\end{tabular}


Table 1. Cont.

\begin{tabular}{|c|c|c|c|c|c|c|c|c|c|c|c|c|c|c|c|}
\hline & \multicolumn{3}{|c|}{ All four PTSD Groups } & \multicolumn{3}{|c|}{ No PTSD Group } & \multicolumn{3}{|c|}{ Delayed PTSD Group } & \multicolumn{3}{|c|}{ Chronic PTSD Group } & \multicolumn{3}{|c|}{$\begin{array}{c}\text { Recovered PTSD } \\
\text { Group }\end{array}$} \\
\hline & No & Yes & Total & No & Yes & Total & No & Yes & Total & No & Yes & Total & No & Yes & Total \\
\hline & $\begin{array}{c}n= \\
3100\end{array}$ & $\begin{array}{c}n= \\
2570\end{array}$ & 5670 & $\begin{array}{c}n= \\
1959\end{array}$ & $\begin{array}{c}n= \\
2138\end{array}$ & 4097 & $\begin{array}{l}n= \\
228\end{array}$ & $\begin{array}{c}n= \\
81\end{array}$ & 309 & $\begin{array}{l}n= \\
341\end{array}$ & $\begin{array}{c}n= \\
65\end{array}$ & 406 & $\begin{array}{l}n= \\
66\end{array}$ & $\begin{array}{l}n= \\
31\end{array}$ & 97 \\
\hline Characteristic & $54.7 \%$ & $45.3 \%$ & & $47.8 \%$ & $52.2 \%$ & & $73.8 \%$ & $26.2 \%$ & & $84.0 \%$ & $16.0 \%$ & & $68.0 \%$ & $32.0 \%$ & \\
\hline \multicolumn{16}{|l|}{ Social Support W4 } \\
\hline \multirow[t]{2}{*}{ Low } & 1027 & 219 & 1246 & 511 & 144 & 655 & 98 & 16 & 114 & 188 & 15 & 203 & 32 & 4 & 36 \\
\hline & 82.4 & 17.6 & & 78.0 & 22.0 & & 86.0 & 14.0 & & 92.6 & 7.4 & & 88.9 & 11.1 & \\
\hline \multirow[t]{2}{*}{ Medium } & 1025 & 604 & 1629 & 657 & 495 & 1152 & 85 & 21 & 106 & 94 & 21 & 115 & 22 & 8 & 30 \\
\hline & 62.9 & 37.1 & & 57.0 & 43.0 & & 80.2 & 19.8 & & 81.7 & 18.3 & & 73.3 & 26.7 & \\
\hline \multirow[t]{2}{*}{ High } & 998 & 1704 & 2702 & 759 & 1465 & 2224 & 42 & 43 & 85 & 51 & 28 & 79 & 12 & 19 & 31 \\
\hline & 36.9 & 63.1 & & 34.1 & 65.9 & & 49.4 & 50.6 & & 64.6 & 35.4 & & 38.7 & 61.3 & \\
\hline \multicolumn{16}{|l|}{$\begin{array}{c}\text { Social Integration } \\
\text { W4 }\end{array}$} \\
\hline \multirow[t]{2}{*}{ low $0-1$} & 200 & 35 & 235 & 81 & 17 & 98 & 23 & 3 & 26 & 54 & 2 & 56 & 3 & 2 & 5 \\
\hline & 85.1 & 14.9 & & 82.7 & 17.4 & & 88.5 & 11.5 & & 96.4 & 3.6 & & 60.0 & 40.0 & \\
\hline \multirow[t]{2}{*}{ Medium 2} & 1379 & 875 & 2254 & 858 & 723 & 1581 & 93 & 28 & 121 & 157 & 21 & 178 & 28 & 11 & 39 \\
\hline & 61.2 & 38.8 & & 54.3 & 45.7 & & 76.9 & 23.1 & & 88.2 & 11.8 & & 71.8 & 28.2 & \\
\hline \multirow[t]{2}{*}{ High 3-4 } & 1477 & 1616 & 3093 & 988 & 1363 & 2351 & 109 & 48 & 157 & 127 & 41 & 168 & 35 & 18 & 53 \\
\hline & 47.8 & 52.3 & & 42.0 & 58.0 & & 69.4 & 30.6 & & 75.6 & 24.4 & & 66.0 & 34.0 & \\
\hline \multicolumn{16}{|l|}{ Self-efficacy W4 } \\
\hline \multirow[t]{2}{*}{ low $<17$} & 1944 & 983 & 2927 & 1042 & 728 & 1770 & 180 & 49 & 229 & 291 & 49 & 340 & 48 & 15 & 63 \\
\hline & 66.4 & 33.6 & & 58.9 & 41.1 & & 78.6 & 21.4 & & 85.6 & 14.4 & & 76.2 & 23.8 & \\
\hline \multirow[t]{2}{*}{ High 17+ } & 1134 & 1570 & 2704 & 905 & 1396 & 2301 & 44 & 31 & 75 & 46 & 16 & 62 & 18 & 16 & 34 \\
\hline & 41.9 & 58.1 & & 39.3 & 60.7 & & 58.7 & 41.3 & & 74.2 & 25.8 & & 52.9 & 47.1 & \\
\hline
\end{tabular}

The multivariable logistic regression results of the psycho-social factors with social embeddedness, stratified by PTSD group are presented in Table 2. High social support was positively associated with social embeddedness, across all four PTSD groups. The no PTSD and chronic PTSD groups showed a statistically significant dose-response relationship with the varying levels of social support. High social integration was significantly associated with social embeddedness for those in the no PTSD $(\mathrm{aOR}=$ adjusted odds ratio; $\mathrm{aOR}=2.23$; 95\% CI: 1.48, 3.36) and chronic PTSD (aOR $=4.93$; 95\% CI: 1.20, 20.26) groups. Social integration was not significantly associated in the delayed or recovered PTSD group. Those exhibiting higher levels of self-efficacy had 1.30 (95\% CI: 1.22, 1.39) times the odds of experiencing social embeddedness in the no PTSD group. Self-efficacy was not statistically significant in the adjusted models for the delayed, chronic, or recovered PTSD groups.

Table 2. Multivariable logistic regression results of the psycho-social factors with social embeddedness.

\begin{tabular}{|c|c|c|c|c|c|c|c|c|c|c|c|c|c|c|c|}
\hline \multirow[b]{3}{*}{ Characteristic } & \multicolumn{3}{|c|}{ All four PTSD Groups } & \multicolumn{3}{|c|}{ No PTSD Group } & \multicolumn{3}{|c|}{ Delayed PTSD Group } & \multicolumn{3}{|c|}{ Chronic PTSD Group } & \multicolumn{3}{|c|}{ Recovered PTSD Group } \\
\hline & \multirow[t]{2}{*}{ aOR } & \multicolumn{2}{|c|}{$95 \% \mathrm{CI}$} & \multirow[t]{2}{*}{ aOR } & \multicolumn{2}{|c|}{$95 \%$ CI } & \multirow[t]{2}{*}{ aOR } & \multicolumn{2}{|c|}{$95 \% \mathrm{CI}$} & \multirow[t]{2}{*}{ aOR } & \multicolumn{2}{|c|}{$95 \% \mathrm{CI}$} & \multirow[t]{2}{*}{ aOR } & \multicolumn{2}{|c|}{$95 \%$ CI } \\
\hline & & Lower & Upper & & Lower & Upper & & Lower & Upper & & Lower & Upper & & Lower & Upper \\
\hline $\begin{array}{c}\text { Marital Status } \\
\text { W1-W4 } \\
\text { Always } \\
\text { Never/sometimes }\end{array}$ & $\begin{array}{c}0.99 \\
\text { ref }\end{array}$ & 0.94 & 1.06 & $\begin{array}{c}0.99 \\
\text { ref }\end{array}$ & 0.93 & 1.06 & 1.01 & 0.68 & 1.51 & $\begin{array}{c}0.81 \\
\text { ref }\end{array}$ & 0.50 & 1.29 & 1.25 & 0.68 & 2.28 \\
\hline $\begin{array}{c}\text { Employment W4 } \\
\text { No } \\
\text { Yes }\end{array}$ & $\begin{array}{c}\text { ref } \\
1.06\end{array}$ & 1.01 & 1.12 & 1.05 & 1.00 & 1.12 & 0.95 & 0.66 & 1.38 & 1.05 & 0.61 & 1.47 & 0.68 & 0.40 & 1.15 \\
\hline $\begin{array}{c}\text { Social Support } \\
\text { W4 } \\
\text { Low } \\
\text { Medium } \\
\text { High }\end{array}$ & $\begin{array}{c}\text { ref } \\
1.95 \\
2.99\end{array}$ & $\begin{array}{l}1.70 \\
2.63\end{array}$ & $\begin{array}{l}2.24 \\
3.41\end{array}$ & $\begin{array}{c}\text { ref } \\
1.86 \\
2.68\end{array}$ & $\begin{array}{l}1.59 \\
2.30\end{array}$ & $\begin{array}{l}2.19 \\
3.12\end{array}$ & $\begin{array}{c}\text { ref } \\
1.30 \\
3.07\end{array}$ & $\begin{array}{l}0.69 \\
1.77\end{array}$ & $\begin{array}{l}2.45 \\
5.35\end{array}$ & $\begin{array}{l}\text { ref } \\
2.27 \\
3.91\end{array}$ & $\begin{array}{l}1.18 \\
2.11\end{array}$ & $\begin{array}{l}4.36 \\
7.23\end{array}$ & $\begin{array}{l}\text { ref } \\
2.87 \\
4.99\end{array}$ & $\begin{array}{l}0.91 \\
1.77\end{array}$ & $\begin{array}{c}9.08 \\
14.03\end{array}$ \\
\hline $\begin{array}{c}\text { Social Integration } \\
\text { W4 } \\
\text { Low 0-1 } \\
\text { Medium } 2 \\
\text { High 3-4 }\end{array}$ & $\begin{array}{c}\text { ref } \\
1.68 \\
2.04 \\
\end{array}$ & $\begin{array}{l}1.25 \\
1.52\end{array}$ & $\begin{array}{l}2.26 \\
2.73\end{array}$ & $\begin{array}{c}\text { ref } \\
1.89 \\
2.23\end{array}$ & $\begin{array}{l}1.25 \\
1.48\end{array}$ & $\begin{array}{l}2.84 \\
3.36\end{array}$ & $\begin{array}{c}\text { ref } \\
1.35 \\
1.52\end{array}$ & $\begin{array}{l}0.42 \\
0.49\end{array}$ & $\begin{array}{l}4.28 \\
4.67\end{array}$ & $\begin{array}{l}\text { ref } \\
2.46 \\
4.93\end{array}$ & $\begin{array}{l}0.57 \\
1.20\end{array}$ & $\begin{array}{l}10.59 \\
20.26\end{array}$ & $\begin{array}{l}\text { ref } \\
0.57 \\
0.72\end{array}$ & $\begin{array}{l}0.13 \\
0.16\end{array}$ & $\begin{array}{l}2.47 \\
3.16\end{array}$ \\
\hline $\begin{array}{c}\text { Self-efficacy W4 } \\
\text { Low }<17 \\
\text { High } 17+\end{array}$ & $\begin{array}{c}\text { ref } \\
1.39\end{array}$ & 1.31 & 1.48 & $\begin{array}{c}\text { ref } \\
1.30\end{array}$ & 1.22 & 1.39 & $\begin{array}{c}\text { ref } \\
1.33\end{array}$ & 0.94 & 1.90 & $\begin{array}{l}\text { ref } \\
1.45\end{array}$ & 0.86 & 2.45 & $\begin{array}{c}\text { ref } \\
1.52\end{array}$ & 0.85 & 2.73 \\
\hline
\end{tabular}

Note: All models are adjusted for age, sex, and race/ethnicity; Bold estimates are statistically significant at the 0.05 level. Ref = reference; $\mathrm{aOR}=$ adjusted odds ratio; $95 \% \mathrm{CI}=95 \%$ confidence interval. 


\subsection{Emotional Embeddedness}

Table 3 shows the distribution of demographic factors and psycho-social factors with emotional embeddedness, stratified by PTSD group. Overall, emotional embeddedness was prevalent among males $(69.6 \%)$, those aged 65 or older $(71.6 \%)$, those who were consistently married $(72.2 \%)$, non-Hispanic Whites $(69.9 \%)$, those who indicated they were employed $(70.8 \%)$, and those with greater levels of social support $(82.6 \%)$, social integration $(73.6 \%)$, and self-efficacy $(83.0 \%)$. The prevalence of emotional embeddedness varied across the PTSD groups. The no PTSD group had the highest prevalence $(68.0 \%)$, followed by the recovered $(41.7 \%)$, delayed (36.5\%), and chronic group (25.7\%).

Table 3. Distribution of demographic factors and psycho-social factors with emotional embeddedness, stratified by PTSD group.

\begin{tabular}{|c|c|c|c|c|c|c|c|c|c|c|c|c|c|c|c|}
\hline & \multicolumn{3}{|c|}{ All four PTSD Groups } & \multicolumn{3}{|c|}{ No PTSD Group } & \multicolumn{3}{|c|}{ Delayed PTSD Group } & \multicolumn{3}{|c|}{ Chronic PTSD Group } & \multicolumn{3}{|c|}{$\begin{array}{c}\text { Recovered PTSD } \\
\text { Group }\end{array}$} \\
\hline & No & Yes & Total & No & Yes & Total & No & Yes & Total & No & Yes & Total & No & Yes & Total \\
\hline & $\begin{array}{c}n= \\
1815\end{array}$ & $\begin{array}{c}n= \\
3851\end{array}$ & 5666 & $\begin{array}{l}n= \\
857\end{array}$ & $\begin{array}{c}n= \\
3239\end{array}$ & 4096 & $\begin{array}{l}n= \\
197\end{array}$ & $\begin{array}{l}n= \\
113\end{array}$ & 310 & $\begin{array}{l}n= \\
298\end{array}$ & $\begin{array}{l}n= \\
103\end{array}$ & 401 & $\begin{array}{c}n= \\
56\end{array}$ & $\begin{array}{c}n= \\
40\end{array}$ & 96 \\
\hline Characteristic & $32.0 \%$ & $68.0 \%$ & & $32.0 \%$ & $68.0 \%$ & & $63.6 \%$ & $36.5 \%$ & & $74.3 \%$ & $25.7 \%$ & & $58.3 \%$ & $41.7 \%$ & \\
\hline \multicolumn{16}{|l|}{ Gender } \\
\hline \multirow[t]{2}{*}{ Male } & 1118 & 2562 & 3680 & 521 & 2190 & 2711 & 138 & 79 & 217 & 172 & 56 & 228 & 31 & 22 & 53 \\
\hline & 30.4 & 69.6 & & 19.2 & 80.8 & & 63.6 & 36.4 & & 75.4 & 24.6 & & 58.5 & 41.5 & \\
\hline \multirow[t]{2}{*}{ Female } & 697 & 1289 & 1986 & 336 & 1049 & 1385 & 59 & 34 & 93 & 126 & 47 & 173 & 25 & 18 & 43 \\
\hline & 35.1 & 64.9 & & 24.3 & 75.7 & & 63.4 & 36.6 & & 72.8 & 27.2 & & 58.1 & 41.9 & \\
\hline \multicolumn{16}{|l|}{$\begin{array}{l}\text { Age on Injury } \\
\text { Survey(years) }\end{array}$} \\
\hline \multirow[t]{2}{*}{$<50$} & 359 & 824 & 1183 & 177 & 699 & 876 & 46 & 27 & 73 & 51 & 11 & 62 & 8 & 9 & 17 \\
\hline & 30.4 & 69.7 & & 20.2 & 79.8 & & 63.0 & 37.0 & & 82.3 & 17.7 & & 47.1 & 52.9 & \\
\hline \multirow[t]{2}{*}{$50-59$} & 649 & 1208 & 1857 & 291 & 987 & 1278 & 80 & 44 & 124 & 115 & 44 & 159 & 21 & 16 & 37 \\
\hline & 35.0 & 65.1 & & 22.8 & 77.2 & & 64.5 & 35.5 & & 72.3 & 27.7 & & 56.8 & 43.2 & \\
\hline \multirow[t]{2}{*}{$60-64$} & 358 & 688 & 1046 & 160 & 571 & 731 & 33 & 24 & 57 & 58 & 19 & 77 & 14 & 11 & 25 \\
\hline & 34.2 & 65.8 & & 21.9 & 78.1 & & 57.9 & 42.1 & & 75.3 & 24.7 & & 56.0 & 44.0 & \\
\hline \multirow[t]{2}{*}{$65+$} & 449 & 1131 & 1580 & 229 & 982 & 1211 & 38 & 18 & 56 & 74 & 29 & 103 & 13 & 4 & 17 \\
\hline & 28.4 & 71.6 & & 18.9 & 81.1 & & 67.9 & 32.1 & & 71.8 & 28.2 & & 76.5 & 23.5 & \\
\hline \multicolumn{16}{|l|}{$\begin{array}{l}\text { Marital Status } \\
\text { W1-W4 }\end{array}$} \\
\hline \multirow{2}{*}{ Always } & 926 & 2401 & 3327 & 452 & 2069 & 2521 & 109 & 64 & 173 & 142 & 43 & 185 & 23 & 22 & 45 \\
\hline & 27.8 & 72.2 & & 17.9 & 82.1 & & 63.0 & 37.0 & & 76.8 & 23.2 & & 51.1 & 48.9 & \\
\hline \multirow[t]{2}{*}{ Never/sometimes } & 889 & 1450 & 2339 & 405 & 1170 & 1575 & 88 & 49 & 137 & 156 & 60 & 216 & 33 & 18 & 51 \\
\hline & 38.0 & 62.0 & & 25.7 & 74.3 & & 64.2 & 35.8 & & 72.2 & 27.8 & & 64.7 & 35.3 & \\
\hline \multicolumn{16}{|l|}{ Race/ethnicity } \\
\hline \multirow{2}{*}{$\begin{array}{l}\text { Non-Hispanic } \\
\text { White }\end{array}$} & 1344 & 3120 & 4464 & 668 & 2660 & 3328 & 148 & 89 & 237 & 188 & 73 & 261 & 37 & 31 & 68 \\
\hline & 30.1 & 69.9 & & 20.1 & 79.9 & & 62.5 & 37.6 & & 72.0 & 28.0 & & 54.4 & 45.6 & \\
\hline $\begin{array}{l}\text { Non-Hispanic } \\
\text { Black }\end{array}$ & 138 & 262 & 400 & 48 & 216 & 264 & 17 & 6 & 23 & 37 & 11 & 48 & 4 & 1 & 5 \\
\hline & 34.5 & 65.5 & & 18.2 & 81.8 & & 73.9 & 26.1 & & 77.1 & 22.9 & & 80.0 & 20.0 & \\
\hline Hispanic & 203 & 266 & 469 & 80 & 194 & 274 & 21 & 12 & 33 & 44 & 13 & 57 & 11 & 7 & 18 \\
\hline & 43.3 & 56.7 & & 29.2 & 70.8 & & 63.6 & 36.4 & & 77.2 & 22.8 & & 61.1 & 38.9 & \\
\hline Asian & 70 & 120 & 190 & 34 & 103 & 137 & 4 & 4 & 8 & 17 & 3 & 20 & 1 & 1 & 2 \\
\hline & 36.8 & 63.2 & & 24.8 & 75.2 & & 50.0 & 50.0 & & 85.0 & 15.0 & & 50.0 & 50.0 & \\
\hline Multiracial/other & 60 & 83 & 143 & 27 & 66 & 93 & 7 & 2 & 9 & 12 & 3 & 15 & 3 & 0 & 3 \\
\hline & 42.0 & 58.0 & & 29.0 & 71.0 & & 77.8 & 22.2 & & 80.0 & 20.0 & & 100.0 & 0.0 & \\
\hline Employment W4 & & & & & & & & & & & & & & & \\
\hline No & 864 & 1541 & 2405 & 349 & 1249 & 1598 & 99 & 56 & 155 & 195 & 58 & 253 & 24 & 12 & 36 \\
\hline & 35.9 & 64.1 & & 21.8 & 78.2 & & 63.9 & 36.1 & & 77.1 & 22.9 & & 66.7 & 33.3 & \\
\hline Yes & 951 & 2310 & 3261 & 508 & 1990 & 2498 & 98 & 57 & 155 & 103 & 45 & 148 & 32 & 28 & 60 \\
\hline & 29.2 & 70.8 & & 20.3 & 79.7 & & 63.2 & 36.8 & & 69.6 & 30.4 & & 53.3 & 46.7 & \\
\hline Social Support W4 & & & & & & & & & & & & & & & \\
\hline Low & 749 & 499 & 1248 & 292 & 366 & 658 & 92 & 21 & 113 & 158 & 44 & 202 & 30 & 6 & 36 \\
\hline & 60.0 & 40.0 & & 44.4 & 55.6 & & 81.4 & 18.6 & & 78.2 & 21.8 & & 83.3 & 16.7 & \\
\hline Medium & 556 & 1068 & 1624 & 277 & 873 & 1150 & 59 & 47 & 106 & 80 & 33 & 113 & 14 & 15 & 29 \\
\hline & 34.2 & 65.8 & & 24.1 & 75.9 & & 55.7 & 44.3 & & 70.8 & 29.2 & & 48.3 & 51.7 & \\
\hline High & 469 & 2232 & 2701 & 266 & 1958 & 2224 & 43 & 44 & 87 & 51 & 25 & 76 & 11 & 19 & 30 \\
\hline & 17.4 & 82.6 & & 12.0 & 88.0 & & 49.4 & 50.6 & & 67.1 & 32.9 & & 36.7 & 63.3 & \\
\hline
\end{tabular}


Table 3. Cont.

\begin{tabular}{|c|c|c|c|c|c|c|c|c|c|c|c|c|c|c|c|}
\hline & \multicolumn{3}{|c|}{ All four PTSD Groups } & \multicolumn{3}{|c|}{ No PTSD Group } & \multicolumn{3}{|c|}{ Delayed PTSD Group } & \multicolumn{3}{|c|}{ Chronic PTSD Group } & \multicolumn{3}{|c|}{$\begin{array}{c}\text { Recovered PTSD } \\
\text { Group }\end{array}$} \\
\hline & No & Yes & Total & No & Yes & Total & No & Yes & Total & No & Yes & Total & No & Yes & Total \\
\hline & $\begin{array}{c}n= \\
1815\end{array}$ & $\begin{array}{c}n= \\
3851\end{array}$ & 5666 & $\begin{array}{l}n= \\
857\end{array}$ & $\begin{array}{c}n= \\
3239\end{array}$ & 4096 & $\begin{array}{l}n= \\
197\end{array}$ & $\begin{array}{l}n= \\
113\end{array}$ & 310 & $\begin{array}{l}n= \\
298\end{array}$ & $\begin{array}{l}n= \\
103\end{array}$ & 401 & $\begin{array}{l}n= \\
56\end{array}$ & $\begin{array}{c}n= \\
40\end{array}$ & 96 \\
\hline Characteristic & $32.0 \%$ & $68.0 \%$ & & $32.0 \%$ & $68.0 \%$ & & $63.6 \%$ & $36.5 \%$ & & $74.3 \%$ & $25.7 \%$ & & $58.3 \%$ & $41.7 \%$ & \\
\hline \multicolumn{16}{|l|}{$\begin{array}{c}\text { Social Integration } \\
\text { W4 }\end{array}$} \\
\hline Low $0-1$ & $\begin{array}{l}158 \\
67.8\end{array}$ & $\begin{array}{c}75 \\
32.2\end{array}$ & 233 & $\begin{array}{c}47 \\
48.5\end{array}$ & $\begin{array}{c}50 \\
51.6\end{array}$ & 97 & $\begin{array}{c}22 \\
84.6\end{array}$ & $\begin{array}{c}4 \\
15.4\end{array}$ & 26 & $\begin{array}{c}49 \\
89.1\end{array}$ & $\begin{array}{c}6 \\
10.9\end{array}$ & 55 & $\begin{array}{c}3 \\
60.0\end{array}$ & $\begin{array}{c}2 \\
40.0\end{array}$ & 5 \\
\hline Medium 2 & $\begin{array}{l}814 \\
36.2\end{array}$ & $\begin{array}{l}1434 \\
63.8\end{array}$ & 2248 & $\begin{array}{l}389 \\
24.7\end{array}$ & $\begin{array}{l}1189 \\
75.4\end{array}$ & 1578 & $\begin{array}{c}81 \\
66.4\end{array}$ & $\begin{array}{c}41 \\
33.6\end{array}$ & 122 & $\begin{array}{l}131 \\
74.0\end{array}$ & $\begin{array}{c}46 \\
26.0\end{array}$ & 177 & $\begin{array}{c}22 \\
59.5\end{array}$ & $\begin{array}{c}15 \\
40.5\end{array}$ & 37 \\
\hline High 3-4 & $\begin{array}{l}817 \\
26.4\end{array}$ & $\begin{array}{l}2280 \\
73.6\end{array}$ & 3097 & $\begin{array}{l}407 \\
17.3\end{array}$ & $\begin{array}{l}1947 \\
82.7\end{array}$ & 2354 & $\begin{array}{c}90 \\
57.3\end{array}$ & $\begin{array}{c}67 \\
42.7\end{array}$ & 157 & $\begin{array}{l}116 \\
70.3\end{array}$ & $\begin{array}{c}49 \\
29.7\end{array}$ & 165 & $\begin{array}{c}31 \\
57.4\end{array}$ & $\begin{array}{c}23 \\
42.6\end{array}$ & 54 \\
\hline Self-efficacy W4 & & & & & & & & & & & & & & & \\
\hline Low <17) & $\begin{array}{l}1337 \\
45.8\end{array}$ & $\begin{array}{l}1585 \\
54.2\end{array}$ & 2922 & $\begin{array}{l}560 \\
31.7\end{array}$ & $\begin{array}{l}1206 \\
68.3\end{array}$ & 1766 & $\begin{array}{l}151 \\
65.9\end{array}$ & $\begin{array}{c}78 \\
34.1\end{array}$ & 229 & $\begin{array}{l}259 \\
76.6\end{array}$ & $\begin{array}{c}79 \\
23.4\end{array}$ & 338 & $\begin{array}{c}41 \\
65.1\end{array}$ & $\begin{array}{c}22 \\
34.9\end{array}$ & 63 \\
\hline High $17+$ & $\begin{array}{l}460 \\
17.0\end{array}$ & $\begin{array}{l}2246 \\
83.0\end{array}$ & 2706 & $\begin{array}{l}288 \\
12.5\end{array}$ & $\begin{array}{l}2017 \\
87.5\end{array}$ & 2305 & $\begin{array}{c}42 \\
55.3\end{array}$ & $\begin{array}{c}34 \\
44.7\end{array}$ & 76 & $\begin{array}{c}35 \\
59.3\end{array}$ & $\begin{array}{c}24 \\
40.7\end{array}$ & 59 & $\begin{array}{c}15 \\
45.5\end{array}$ & $\begin{array}{c}18 \\
55.5\end{array}$ & 33 \\
\hline
\end{tabular}

Table 4 presents the multivariable logistic regression results of the psycho-social factors with emotional embeddedness by each PTSD group. Social support showed a dose-response relationship with medium and high levels increasing the odds of emotional embeddedness in the no PTSD, delayed, and recovered groups. Social support was not statistically significant among those in the chronic PTSD group. High social integration was significantly associated with emotional embeddedness in the no PTSD $(a O R=1.35 ; 95 \%$ CI: 1.11, 1.63) group. High self-efficacy was also significantly associated with emotional embeddedness in the no PTSD (aOR $=1.21 ; 95 \%$ CI: $1.17,1.25)$ and chronic $(\mathrm{aOR}=1.62$; $95 \%$ CI: 1.12, 2.35) groups. Social integration and self-efficacy were not associated with emotional embeddedness in the delayed or recovered groups.

Table 4. Multivariable logistic regression results of the psycho-social factors with emotional embeddedness, stratified by PTSD group.

\begin{tabular}{|c|c|c|c|c|c|c|c|c|c|c|c|c|c|c|c|}
\hline \multirow[b]{3}{*}{ Characteristic } & \multirow{3}{*}{$\begin{array}{l}\text { All } \\
\text { Four } \\
\text { PTSD } \\
\text { Groups } \\
\text { aOR } \\
\end{array}$} & & & \multicolumn{3}{|c|}{ No PTSD Group } & \multicolumn{3}{|c|}{ Delayed PTSD Group } & \multicolumn{3}{|c|}{ Chronic PTSD Group } & \multicolumn{3}{|c|}{ Recovered PTSD Group } \\
\hline & & \multicolumn{2}{|c|}{$95 \% \mathrm{CI}$} & aOR & \multicolumn{2}{|c|}{$95 \%$ CI } & aOR & \multicolumn{2}{|c|}{$95 \% \mathrm{CI}$} & aOR & \multicolumn{2}{|c|}{$95 \% \mathrm{CI}$} & aOR & \multicolumn{2}{|c|}{$95 \%$ CI } \\
\hline & & Lower & Upper & & Lower & Upper & & Lower & Upper & & Lower & Upper & & Lower & Upper \\
\hline $\begin{array}{c}\text { Marital Status } \\
\text { W1-W4 } \\
\text { Always } \\
\text { Never/sometimes }\end{array}$ & $\begin{array}{c}1.00 \\
\text { ref }\end{array}$ & 0.96 & 1.04 & $\begin{array}{c}0.99 \\
\text { ref }\end{array}$ & 0.96 & 1.03 & $\begin{array}{c}1.25 \\
\text { ref }\end{array}$ & 0.93 & 1.68 & $\begin{array}{c}1.33 \\
\text { ref }\end{array}$ & 0.93 & 1.92 & $\begin{array}{l}1.05 \\
\text { ref }\end{array}$ & 0.68 & 1.63 \\
\hline $\begin{array}{c}\text { Employment } \\
\text { W4 } \\
\text { No } \\
\text { Yes }\end{array}$ & $\begin{array}{c}\text { ref } \\
1.09 \\
\end{array}$ & 1.05 & 1.13 & $\begin{array}{c}\text { ref } \\
1.03\end{array}$ & 0.99 & 1.06 & $\begin{array}{c}\text { ref } \\
1.06\end{array}$ & 0.80 & 1.40 & $\begin{array}{c}\text { ref } \\
1.18\end{array}$ & 0.85 & 1.65 & $\begin{array}{c}\text { ref } \\
1.32\end{array}$ & 0.78 & 2.24 \\
\hline $\begin{array}{c}\text { Social Support } \\
\text { W4 } \\
\text { Low } \\
\text { Medium }\end{array}$ & $\begin{array}{c}\text { ref } \\
1.55\end{array}$ & 1.44 & 1.67 & $\begin{array}{c}\text { ref } \\
1.33\end{array}$ & 1.23 & 1.43 & $\begin{array}{c}\text { ref } \\
2.63\end{array}$ & 1.67 & 4.15 & $\begin{array}{c}\text { ref } \\
\mathbf{1 . 3 2}\end{array}$ & 0.88 & 1.97 & $\begin{array}{c}\text { ref } \\
3.34\end{array}$ & 1.49 & 7.49 \\
\hline High & 1.78 & 1.66 & 1.92 & 1.47 & 1.37 & 1.58 & 2.88 & 1.82 & 4.54 & 1.42 & 0.92 & 2.20 & 3.62 & 1.60 & 8.20 \\
\hline $\begin{array}{c}\text { Social } \\
\text { Integration W4 } \\
\text { Low 0-1 } \\
\text { Medium 2 } \\
\text { High 3-4 }\end{array}$ & $\begin{array}{c}\text { ref } \\
1.53 \\
1.66\end{array}$ & $\begin{array}{l}1.28 \\
1.38\end{array}$ & $\begin{array}{l}1.84 \\
1.99\end{array}$ & $\begin{array}{c}\text { ref } \\
1.27 \\
1.35\end{array}$ & $\begin{array}{l}1.05 \\
1.11\end{array}$ & $\begin{array}{l}1.54 \\
1.63\end{array}$ & $\begin{array}{c}\text { ref } \\
1.92 \\
2.04\end{array}$ & $\begin{array}{l}0.74 \\
0.80\end{array}$ & $\begin{array}{l}4.99 \\
5.19\end{array}$ & $\begin{array}{c}\text { ref } \\
1.80 \\
2.10\end{array}$ & $\begin{array}{l}0.80 \\
0.93\end{array}$ & $\begin{array}{l}4.07 \\
4.73\end{array}$ & $\begin{array}{c}\text { ref } \\
1.11 \\
1.17\end{array}$ & $\begin{array}{l}0.38 \\
0.41\end{array}$ & $\begin{array}{l}3.21 \\
3.32\end{array}$ \\
\hline $\begin{array}{c}\text { Self-efficacy W4 } \\
\text { Low }<17 \\
\text { High } 17+\end{array}$ & $\begin{array}{c}\text { ref } \\
1.35\end{array}$ & 1.30 & 1.40 & $\begin{array}{c}\text { ref } \\
1.21\end{array}$ & 1.17 & 1.25 & $\begin{array}{c}\text { ref } \\
1.16\end{array}$ & 0.86 & 1.56 & $\begin{array}{c}\text { ref } \\
\mathbf{1 . 6 2}\end{array}$ & 1.12 & 2.35 & $\begin{array}{c}\text { ref } \\
1.20\end{array}$ & 0.72 & 2.01 \\
\hline
\end{tabular}

Note: All models are adjusted for age, sex, and race/ethnicity; bold estimates are statistically significant at the 0.05 level. Ref $=$ reference; $\mathrm{aOR}=$ adjusted odds ratio; $95 \% \mathrm{CI}=95 \%$ confidence interval. 


\section{Discussion}

As was hypothesized, persons who had chronic PTSD had the lowest prevalence of both social and emotional embeddedness and over half of persons with no symptoms indicative of PTSD following 9/11 reported social and emotional embeddedness. Only a slightly higher proportion of those who "recovered" indicated social and emotional embeddedness than the delayed group. Surprisingly, the prevalence of emotional embeddedness was higher than social embeddedness for all PTSD strata, suggesting the importance of addressing the emotional needs of $9 / 11$ survivors, in addition to their social needs. More nuanced findings for PTSD status were found, suggesting that social embeddedness was greater among the no PTSD and chronic PTSD status, and social integration was higher for the recovered or delayed PTSD status.

Critically, research tends to focus on the social aspects of loneliness or isolation and how these factors contribute to one's well-being [36-38], while gerontological studies tend to focus on the social connections individuals develop and maintain during their later years and how their presence contributes to their health [39-41]. Further, the associations between loneliness and isolation, and their antithesis of embeddedness, have not previously been demonstrated with regard to the potential associations with PTSD. Given the abundance of literature on PTSD and its potential correlates for physical health functioning among trauma-exposed populations this was a particularly important facet of this study, which is the first to address the concepts of emotional and social embeddedness and their associations with PTSD among a 9/11-exposed population to our knowledge.

Findings from this study suggest that individuals who reported experiencing no symptoms of PTSD reported greater emotional and social embeddedness, whereas those who reported symptoms of PTSD, whether chronic, delayed, or recovered, were less likely to do so. Thus, these findings further support the notion that the social and emotional barriers often coupled with symptoms of PTSD are likely to detrimentally influence one's ability and willingness to engage in socially or emotionally embedding situations (e.g., attend social events). Through identifying these connections, this study helps to better expand on potential factors that may ultimately improve social and emotional functioning for those individuals experiencing symptoms of PTSD (e.g., social activities, emotional connections/supports). Among those enrollees who ever experienced PTSD symptoms, we found that the recovered group tended to report the highest levels of social and emotional embeddedness, followed by the delayed group, with the lowest prevalence reported by the chronic group. Moreover, it appears that the prevalence of social embeddedness was less among those individuals who experienced PTSD symptoms, compared with emotional embeddedness, which could be attributed to a variety of factors including sociodemographic, neighborhood/community, access, and focus of treatment. Further, it is possible that emotional embeddedness in contrast to social embeddedness may play a more prominent role in abating symptoms of PTSD among those who experienced or continue to experience PTSD after an exposure to a traumatic event. Importantly, future research would need to evaluate whether social or emotional embeddedness precedes or is a result of a reduction in PTSD symptoms. Critically, we must keep in mind that regardless of PTSD status, the experiences of embeddedness, and the alternative, loneliness, are subjective and are hard to measure and track over time [42]. It is also possible that those enrollees who reported delayed or chronic PTSD may have experienced additional traumas post-9/11 that were not captured in this study. Specifically, data on traumas post-9/11 were only captured during Wave 4 and when included in our models, did not change the strength of the associations.

Another explanation of less social than emotional embeddedness among persons in the PTSD recovery group is that social embeddedness implies an outward nature of acting or engaging with others. On the one hand, those who did not report experiencing any PTSD symptoms would be more likely to report social embeddedness given the lack of psychological barriers associated with PTSD. On the other hand, individuals who report PTSD symptoms may experience varying degrees of psychological barriers, in turn 
impacting the ability to engage in more socially driven activities. As evidenced in a sample of Vietnam veterans, those with chronic PTSD may become more accustomed to the nature of the disorder and choose to avoid social situations in order to reduce the likelihood of being triggered [43]. As such, future studies may benefit from considering whether PTSD symptom clusters among those who express greater avoidance symptoms would, in fact, report diminished embeddedness.

Other factors may also contribute to the experience of social embeddedness among the recovered group. For instance, individuals who no longer experience symptoms of PTSD may have received additional therapeutic interventions that increased their feelings of selfefficacy, in turn encouraging them to engage in more socially oriented ways. In fact, among persons with no PTSD symptoms who reported higher levels of self-efficacy, we found they were 1.3 times as likely to report social embeddedness compared with those in the delayed, recovered, and chronic PTSD group (see Table 3). This suggests that the mere presence of PTSD symptoms, whether enduring or not, may diminish one's sense of self-efficacy, and in turn, reduce one's ability to experience more social embeddedness. Garrey et al. [44] found that those individuals who sought treatment had greater self-efficacy than those not in treatment for PTSD. Conversely, it may also be that people with low self-efficacy could be more likely to experience PTSD.

As indicated, all four of the groups in this study reported a higher prevalence of emotional embeddedness compared with social embeddedness. One potential reason for this difference may be related to how emotional embeddedness reflects a more internal or intrinsic experience and perspective. As opposed to engaging with others socially, emotional embeddedness allows for individuals to become "close" to individuals without the need for physical proximity. In fact, studies suggest that the interactions one has with others may be less critical for developing meaningful relationships; rather, the quality of the relationship and emotional connection may be more salient [45].

\section{Limitations}

Despite the many strengths of this study, several limitations must be mentioned. First, it is important to note that although we attempted to include as many enrollees as possible in our analyses, our PTSD groups were not all equal in size. Due to this, it is possible that some of the smaller sample-sized PTSD groups were not as sufficiently powered and our ability to detect true effect sizes was diminished. Importantly, these sample size differences are typically the product of enrollees being lost to follow-up and not to other factors within the control of the researchers. Further, although the de Jong Gierveld 6-item loneliness measure [28] used in this study has been validated in a previous study, our specific scoring strategies and usage of the scale as a reverse measure of embeddedness has not been validated. Future studies should attempt to replicate these findings using these measurement strategies to validate the findings and confirm their usage in this manner. As mentioned in the discussion, individuals who reported delayed or chronic PTSD may have done so due to additional traumatic experiences they had post-9/11. Given our study only captured additional traumas post- $9 / 11$ at one time point, it would be beneficial for future studies to gather this information at multiple time points to assess the impact of traumatic exposure, as well as control for extraneous impacts, including history effects, post-9/11. It may also be beneficial to expand upon the quantitative data collection strategies by incorporating a qualitative design to better understand the underlying factors driving feelings or experiences of embeddedness. Critically, given this survey was conducted 15 years post-9/11, the causal implications of PTSD and embeddedness are not able to be determined. Despite our efforts to gather PTSD information across all Waves of the Registry surveys, the HQoL survey was only conducted once. As such, it may be that embeddedness may potentially be a consequence of PTSD, as opposed to a driving mechanism. Future studies would benefit from addressing this potential additional consideration, as well as gathering embeddedness information over time. Finally, although the Registry possesses many benefits it is important to note that it is a closed cohort of individuals with data 
collection periods often spanning large intervals of time. Therefore, it is difficult for us to fully determine the timing of PTSD group membership and when/if movement from one group to another has occurred. Future research would benefit from further addressing these PTSD groupings to determine the static and dynamic nature of their classifications and what factors, including those of emotional and social embeddedness, may contribute to or thwart these transitions.

\section{Conclusions}

This study assessed the prevalence and association of social embeddedness and psychosocial factors by PTSD status for survivors and first responders of 9/11. Our findings suggest that those individuals who report little to no PTSD also report the most social/emotional embeddedness; whereas those individuals who report greater or chronic PTSD report the least social/emotional embeddedness. To better address the nature of social and emotional embeddedness among those individuals who report symptoms of PTSD, clinicians across professional contexts should address the social lives of these individuals and ensure their emotional and physical needs are being met.

Author Contributions: Conceptualization, R.M.B., M.K.H., C.D.P., S.O., S.K.G., F.M.O. and A.S.; formal analysis, C.D.P. and S.O.; methodology, M.K.H. and R.M.B.; project administration, M.K.H.; writing, M.K.H., C.D.P. and R.M.B.; writing-reviewing and editing, R.M.B., M.K.H., C.D.P., S.O., S.K.G., F.M.O. and A.S. All authors have read and agreed to the published version of the manuscript.

Funding: This study was supported by Cooperative Agreement U50/ATU272750 from the Agency for Toxic Substances and Disease Registry (ATSDR) and the Centers for Disease Control and Prevention (CDC), which included support from the National Center for Environmental Health and by Cooperative Agreement U50/OH009739 from the National Institute for Occupational Safety and Health (NIOSH) and the New York City Department of Health and Mental Hygiene. The findings and conclusions in this report are those of the authors and do not necessarily represent the views of the ATSDR or CDC/NIOSH.

Institutional Review Board Statement: The study was conducted according to the guidelines of the Declaration of Helsinki, and approved by the Institutional Review Board of New York City Department of Health and Mental Hygiene (Registry protocol number is 02058 and most recent date of approval is 5/11/2021; HQoL protocol number is $17-084$ which was deemed exempt on 23 June 2017).

Informed Consent Statement: Informed consent was obtained from all subjects involved in this study.

Data Availability Statement: This data is not publicly available. Requests for this or other Registry data can be sent to rbrackbi@health.nyc.gov.

Conflicts of Interest: The authors declare no conflict of interest.

\section{References}

1. Adams, S.W.; Bowler, R.M.; Russell, K.; Brackbill, R.M.; Li, J.; Cone, J.E. PTSD and comorbid depression: Social support and self-efficacy in World Trade Center tower survivors 14-15 years after 9/11. Psychol. Trauma Theory Res. Pract. Policy 2019, 11, 156-164. [CrossRef] [PubMed]

2. DiGrande, L.; Perrin, M.A.; Thorpe, L.E.; Thalji, L.; Murphy, J.; Wu, D.; Farfel, M.; Brackbill, R.M. Posttraumatic stress symptoms, PTSD, and risk factors among lower Manhattan residents 2-3 years after the September 11, 2001 terrorist attacks. J. Trauma. Stress Off. Publ. Int. Soc. Trauma. Stress Stud. 2008, 21, 264-273. [CrossRef] [PubMed]

3. Brackbill, R.M.; Thorpe, L.E.; DiGrande, L.; Perrin, M.; Sapp, J.H.; Wu, D.; Campolucci, S.; Walker, D.J.; Cone, J.; Pulliam, P. Surveillance for World Trade Center disaster health effects among survivors of collapsed and damaged buildings. Morb. Mortal. Wkly. Rep. Surveill. Summ. 2006, 55, 1-18.

4. Caramanica, K.; Brackbill, R.M.; Liao, T.; Stellman, S.D. Comorbidity of 9/11-Related PTSD and Depression in the World Trade Center Health Registry 10-11 Years Postdisaster. J. Trauma. Stress 2014, 27, 680-688. [CrossRef]

5. Brackbill, R.M.; Stellman, S.D.; Perlman, S.E.; Walker, D.J.; Farfel, M.R. Mental health of those directly exposed to the World Trade Center disaster: Unmet mental health care need, mental health treatment service use, and quality of life. Soc. Sci. Med. 2013, 81, 110-114. [CrossRef] 
6. Hamwey, M.K.; Gargano, L.M.; Friedman, L.G.; Leon, L.F.; Petrsoric, L.J.; Brackbill, R.M. Post-Traumatic Stress Disorder among Survivors of the September 11, 2001 World Trade Center Attacks: A Review of the Literature. Int. J. Environ. Res. Public Health 2020, 17, 4344. [CrossRef]

7. Zatzick, D.; Russo, J.; Grossman, D.C.; Jurkovich, G.; Sabin, J.; Berliner, L.; Rivara, F. Posttraumatic stress and depressive symptoms, alcohol use, and recurrent traumatic life events in a representative sample of hospitalized injured adolescents and their parents. J. Pediatr. Psychol. 2006, 31, 377-387. [CrossRef]

8. Schnurr, P.P.; Lunney, C.A.; Bovin, M.J.; Marx, B.P. Posttraumatic stress disorder and quality of life: Extension of findings to veterans of the wars in Iraq and Afghanistan. Clin. Psychol. Rev. 2009, 29, 727-735. [CrossRef]

9. Cone, J.E.; Li, J.; Kornblith, E.; Gocheva, V.; Stellman, S.D.; Shaikh, A.; Schwarzer, R.; Bowler, R.M. Chronic probable PTSD in police responders in the world trade center health registry ten to eleven years after 9/11. Am. J. Ind. Med. 2015, 58, 483-493. [CrossRef]

10. Laffaye, C.; Cavella, S.; Drescher, K.; Rosen, C. Relationships among PTSD symptoms, social support, and support source in veterans with chronic PTSD. J. Trauma. Stress Off. Publ. Int. Soc. Trauma. Stress Stud. 2008, 21, 394-401. [CrossRef]

11. Leigh-Hunt, N.; Bagguley, D.; Bash, K.; Turner, V.; Turnbull, S.; Valtorta, N.; Caan, W. An overview of systematic reviews on the public health consequences of social isolation and loneliness. Public Health 2017, 152, 157-171. [CrossRef]

12. Nicholson, N.R., Jr.; Feinn, R.; Casey, E.; Dixon, J. Psychometric evaluation of the social isolation scale in older adults. Gerontologist 2020, 60, e491-e501. [CrossRef]

13. Eng, P.M.; Rimm, E.B.; Fitzmaurice, G.; Kawachi, I. Social ties and change in social ties in relation to subsequent total and cause-specific mortality and coronary heart disease incidence in men. Am. J. Epidemiol. 2002, 155, 700-709. [CrossRef]

14. Cacioppo, J.T.; Hughes, M.E.; Waite, L.J.; Hawkley, L.C.; Thisted, R.A. Loneliness as a specific risk factor for depressive symptoms: Cross-sectional and longitudinal analyses. Psychol. Aging 2006, 21, 140. [CrossRef]

15. Goldsmith, S.K.; Pellmar, T.C.; Kleinman, A.M.; Bunney, W.E. Reducing Suicide: A National Imperative; National Academies Press: Washington, DC, USA, 2002.

16. Teo, A.R.; Marsh, H.E.; Forsberg, C.W.; Nicolaidis, C.; Chen, J.I.; Newsom, J.; Saha, S.; Dobscha, S.K. Loneliness is closely associated with depression outcomes and suicidal ideation among military veterans in primary care. J. Affect. Disord. 2018, 230, 42-49. [CrossRef]

17. Cacioppo, S.; Grippo, A.J.; London, S.; Goossens, L.; Cacioppo, J.T. Loneliness: Clinical import and interventions. Perspect. Psychol. Sci. 2015, 10, 238-249. [CrossRef]

18. Cacioppo, J.T.; Berntson, G.G.; Larsen, J.T.; Poehlmann, K.M.; Ito, T.A. The psychophysiology of emotion. Handb. Emot. 2000, 2, 173-191.

19. Snowden, L.R. Social embeddedness and psychological well-being among African Americans and Whites. Am. J. Community Psychol. 2001, 29, 519-536. [CrossRef]

20. Fang, S.; Wang, X.Q.; Yang, B.X.; Liu, X.J.; Morris, D.L.; Yu, S.H. Survey of Chinese persons managing depressive symptoms: Help-seeking behaviours and their influencing factors. Compr. Psychiatry 2019, 95, 152127. [CrossRef]

21. Teguo, M.T.; Simo-Tabue, N.; Stoykova, R.; Meillon, C.; Cogne, M.; Amiéva, H.; Dartigues, J.-F. Feelings of loneliness and living alone as predictors of mortality in the elderly: The PAQUID study. Psychosom. Med. 2016, 78, 904-909. [CrossRef]

22. Antonucci, T.C.; Ajrouch, K.J.; Birditt, K.S. The convoy model: Explaining social relations from a multidisciplinary perspective. Gerontologist 2014, 54, 82-92. [CrossRef]

23. Benight, C.C.; Bandura, A. Social cognitive theory of posttraumatic recovery: The role of perceived self-efficacy. Behav. Res. Ther. 2004, 42, 1129-1148. [CrossRef]

24. Van der Velden, P.G.; Oudejans, M.; Das, M.; Bosmans, M.W.; Maercker, A. The longitudinal effect of social recognition on PTSD symptomatology and vice versa: Evidence from a population-based study. Psychiatry Res. 2019, 279, 287-294. [CrossRef]

25. Platt, J.M.; Lowe, S.R.; Galea, S.; Norris, F.H.; Koenen, K.C. A longitudinal study of the bidirectional relationship between social support and posttraumatic stress following a natural disaster. J. Trauma. Stress 2016, 29, 205-213. [CrossRef] [PubMed]

26. Pietrzak, R.H.; Feder, A.; Singh, R.; Schechter, C.B.; Bromet, E.J.; Katz, C.; Reissman, D.; Ozbay, F.; Sharma, V.; Crane, M. Trajectories of PTSD risk and resilience in World Trade Center responders: An 8-year prospective cohort study. Psychol. Med. 2014, 44, 205-219. [CrossRef] [PubMed]

27. Welch, A.E.; Caramanica, K.; Maslow, C.B.; Brackbill, R.M.; Stellman, S.D.; Farfel, M.R. Trajectories of PTSD Among Lower Manhattan Residents and Area Workers Following the 2001 World Trade Center Disaster, 2003-2012. J. Trauma. Stress 2016, 29, 158-166. [CrossRef] [PubMed]

28. Farfel, M.; DiGrande, L.; Brackbill, R.; Prann, A.; Cone, J.; Friedman, S.; Walker, D.J.; Pezeshki, G.; Thomas, P.; Galea, S. An overview of $9 / 11$ experiences and respiratory and mental health conditions among World Trade Center Health Registry enrollees. J. Urban Health 2008, 85, 880-909. [CrossRef] [PubMed]

29. De Jong Gierveld, J.; Tilburg, T.V. A 6-item scale for overall, emotional, and social loneliness: Confirmatory tests on survey data. Res. Aging 2006, 28, 582-598. [CrossRef]

30. Blanchard, E.B.; Jones-Alexander, J.; Buckley, T.C.; Forneris, C.A. Psychometric properties of the PTSD Checklist (PCL). Behav. Res. Ther. 1996, 34, 669-673. [CrossRef]

31. Weathers, F.W.; Litz, B.T.; Herman, D.; Huska, J.; Keane, T. The PTSD Checklist-Civilian Version (PCL-C); National Center for PTSD: Boston, MA, USA, 1994; Volume 10. 
32. Association, A.P. Diagnostic Criteria from dsM-iV-tr; American Psychiatric Pub: Washington, DC, USA, 2000.

33. Donald, C.A.; Ware, J.E. The measurement of social support. Res. Community Ment. Health 1984, 4, 325-370.

34. Sherbourne, C.D.; Stewart, A.L. The MOS social support survey. Soc. Sci. Med. 1991, 32, 705-714. [CrossRef]

35. Schwarzer, R.; Jerusalem, M. Generalized self-efficacy scale. In Measures in Health Psychology: A User's Portfolio. Causal and Control Beliefs; NFER-NELSON: Windsor, UK, 1995; Volume 1, pp. 35-37.

36. Schrempft, S.; Jackowska, M.; Hamer, M.; Steptoe, A. Associations between social isolation, loneliness, and objective physical activity in older men and women. BMC Public Health 2019, 19, 74. [CrossRef]

37. Yu, B.; Steptoe, A.; Niu, K.; Ku, P.-W.; Chen, L.-J. Prospective associations of social isolation and loneliness with poor sleep quality in older adults. Qual. Life Res. 2018, 27, 683-691. [CrossRef]

38. De Jong Gierveld, J.; Van Tilburg, T.; Dykstra, P.A. Loneliness and social isolation. In The Cambridge Handbook of Personal Relationships; Perlman, D., Vangelisti, A., Eds.; Cambridge University Press: New York, NY, USA, 2006; pp. 485-500.

39. Luong, G.; Charles, S.T.; Fingerman, K.L. Better with age: Social relationships across adulthood. J. Soc. Pers. Relatsh. 2011, 28, 9-23. [CrossRef]

40. Wagner, M.; Schütze, Y.; Lang, F.R. Social Relationships in Old Age; Cambridge University Press: New York, NY, USA, 1999.

41. Russell, D.; Cutrona, C.E.; Rose, J.; Yurko, K. Social and emotional loneliness: An examination of Weiss's typology of loneliness. J. Personal. Soc. Psychol. 1984, 46, 1313. [CrossRef]

42. Bruine de Bruin, W.; Parker, A.M.; Strough, J. Age differences in reported social networks and well-being. Psychol. Aging 2020, 35, 159. [CrossRef]

43. Orsillo, S.M.; Heimberg, R.G.; Juster, H.R.; Garrett, J. Social phobia and PTSD in Vietnam veterans. J. Trauma. Stress 1996, 9, 235-252.

44. Garrey, S.K.; Welch, A.E.; Jacobson, M.H.; Brackbill, R.M.; Gargano, L.M. The intentional self-medication of 9/11-related PTSD symptoms with alcohol: 15 years after the disaster. Int. J. Environ. Res. Public Health 2020, 17, 5327. [CrossRef]

45. Chang, P.-J.; Wray, L.; Lin, Y. Social relationships, leisure activity, and health in older adults. Health Psychol. $2014,33,516$. [CrossRef] 